\title{
Der Triptanwirkung auf der visuellen Spur
}

Fragestellung: Die vorliegende Untersuchung vergleicht die Effekte von Sumatriptan, Kochsalz und Acetylsalicylsäure (ASS) auf die BOLD-Antwort (Blood oxygen level-depend-Response) nach experimentellen Schmerzreizen.

Hintergrund: Triptane wirken als Agonisten an $5-\mathrm{HT}_{1 \mathrm{~B} / \mathrm{D}^{-}}$ Rezeptoren. Der Mechanismus führt am 5-HT $\mathrm{H}_{1 \mathrm{~B}}$-Rezeptor zu gering ausgeprägter Vasokonstriktion intrakranieller Gefäße und zur Hemmung der Freisetzung von CGRP (Calcitonin Gene-Related Peptide). Zentral im Bereich des Hirnstamms am 5- $\mathrm{HT}_{1 \mathrm{D}^{-}}$ Rezeptor wird die Signaltransduktion im trigeminalen Kerngebiet gehemmt. Im Tiermodell wurden auch inhibitorische Effekte auf die Weiterleitung des Schmerzsignals in thalamischen Kerngebieten gezeigt. Nur in wenigen Studien wurde bisher versucht, bildgebend die Wirkung der Triptane nachzuvollziehen.

Patienten und Methodik: In der randomisierten, placebokontrollierten dopppelblinden funktionellen MRT-Untersuchung (fMRT) wurden 43 gesunde

Kröger IL, May A. Triptan-induced disruption of trigeminocortical connectivity. Neurology 2015; 84: 2124-31
Erwachsene zweimal im Abstand von acht bis 14 Tagen untersucht. Die Sumatriptangruppe umfasste 21, die ASS-Gruppe 22 Patienten.
Während der ersten fMRT-Untersuchung erhielt die Hälfte der Probanden jeder Gruppe Kochsalz, zur zweiten fMRT-Untersuchung wurden die korrespondierende Substanz oder Placebo appliziert. Für die fMRT-Untersuchung wurde eine abgewandelte Form des von der Gruppe etablierten Stimulationsparadigma [1] verwendet. Hierbei kommen Geruchsreize zum Einsatz, als Kontrollbedingung dient ein visueller Stimulus. Die Reizintensität (Schmerz) und die Bewertung als unangenehm wurden auf einer visuellen Analogskala beurteilt.

Ergebnisse: Die Schmerzintensität wurde in allen drei Gruppen (Sumatriptan, ASS und Placebo) gleich bewertet. Die nozizeptiven trigeminalen Stimuli führten zu signifikanten Veränderungen der BOLD-Antwort in kortikalen und subkortikalen Arealen sowie im Hirnstamm und Cerebellum. Nach Sumatriptan wurde im Vergleich zu Placebo eine vermehrte Aktivierung trigeminaler Kerne beobachtet, eine vermehrte BOLDAntwort im Vergleich zu ASS wurde im Thalamus gesehen.

Schlussfolgerungen: Die Ergebnisse bieten durch den Nachweis spezifischer funktioneller triptanvermittelter Hemmung der trigeminokortikalen Verbindungen einen Erklärungsansatz dafür, dass Triptane spezifisch beim Kopfschmerz (insbesondere der Migräne) und nicht bei Schmerzen allgemein wirksam sind.

\section{- Kommentar von Charly Gaul, Königstein}

\section{Ein weiteres Puzzelteil im Verständnis der Triptanwirkung}

Die Studie zeigte eine vermehrte Antwort im Bereich der trigeminalen Kerne und des Thalamus nach Gabe von Sumatriptan, die sich nach ASS nicht zeigt. Dies steht in einem scheinbaren Widerspruch zu Tierversuchen, die einen inhibitorischen Effekt von Sumatriptan auf trigeminovaskuläre Neurone zeigten. Die BOLD-Antwort könnte aber ebenso gut auf eine Aktivierung inhibitorischer Neurone zurückzuführen sein und so den Widerspruch auflösen. Eine solche verstärkte BOLD-Antwort könnte spezifisch für die nozizeptive Transmission zwischen Trigeminus und Thalamus sein, da sie bei den nicht nozizeptiven Stimuli nicht beobachtet wurde. Ein rein antinozizeptiver Effekt kann die Studienergebnisse nicht erklären, da die Veränderungen unter der Gabe von ASS nicht auftraten.

Die Studie zeigte, dass die Verbindung zwischen trigeminalen Kernen und Thalamus, die in der nozizeptiven Transmission relevant ist, durch Sumatriptan beeinflusst werden kann. Einschränkend muss jedoch festgehalten werden, dass die Untersuchung an gesunden Probanden ohne Migräne durchgeführt wurde und bei Patienten mit Migräne Prozesse der zentralen Sensitisierung durchaus zu anderen Ergebnissen führen können. Sicherlich ist es ein nächster Schritt, dass jetzt etablierte Modell mit Migränepatienten zu untersuchen. Die Studie trägt zum Verständnis der spezifischen Triptanwirkung im Vergleich zur unspezifischen Wirkung von ASS wesentlich bei. Dass die
Medikation keinen Einfluss auf die Bewertung der Schmerzintensität hatte, überrascht jedoch sehr, da es sich um ein Modell zur Untersuchung trigeminaler Schmerzreize handelt. Peter Goadsby merkt in einem Kommentar an, der mit der Studie publiziert wurde [2], dass die Ergebnisse nicht nur Fragen beantworten, sondern ebenso viele neue aufwerfen. Er gehe davon aus, dass mittels funktioneller Bildgebung letztlich auch neue Therapieansätze entwickelt werden können.
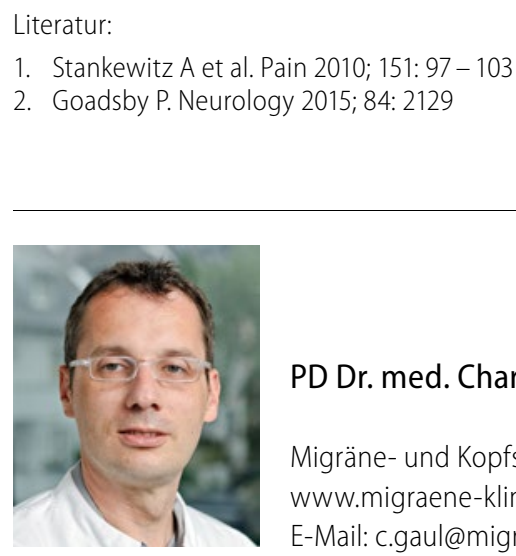

PD Dr. med. Charly Gaul, Königstein

Migräne- und Kopfschmerzklinik Königstein, www.migraene-klinik.de/ E-Mail: c.gaul@migraene-klinik.de 